Therapieempfehlung für Patienten

\section{Würden Sie für sich selbst genauso entscheiden?}

— Ein Patient hat die schwierige Wahl zwischen zwei Behandlungsalternativen: Die Therapie, mit der seine Überlebenschancen höher sind, kann unangenehme Nebenwirkungen zur Folge haben. Wozu würden Sie als Arzt ihm raten? Und welche Behandlung würden Sie wählen, wenn Sie selbst erkrankt wären?

Das ist offenbar nicht immer dasselbe, wie die Befragung von 750 Hausärzten ergab [Ubel PA et al. Arch Intern Med 2011; 171: 630-34]. Bei einem Kolonkarziom würden 37,8\% der Ärzte für sich selbst die Behandlung mit der höheren Mortalität wählen, aber nur $24,5 \%$ würden diese Behandlung ihren Patienten empfehlen. Auch im Fall einer Vogelgrippe nannten die Ärzte das prognostisch weniger günstige Medikament häufiger für die Selbsttherapie (62,9\% vs. $48,5 \%)$.

de

Sinkende Arzneimittelausgaben

\section{Neue Sparinstrumente greifen}

— Nach einem marginalen Zuwachs zu Beginn des Jahres sind die Ausgaben der gesetzlichen Kassen für Arzneimittel und Impfstoffe im Februar wieder deutlich gesunken. Sie lagen um 3,5\% unter dem Vergleichswert des Vorjahresmonats, teilte das Beratungsunternehmen IMS Health mit. Insgesamt haben Vertragsärzte im Februar Arzneimittel und Impfstoffe im Wert von 2,31 Mrd. Euro verordnet. Für die ersten beiden Monate des Jahres ergeben sich damit Gesamtausgaben von 4,74 Mrd. Euro. Das entspricht einem Rückgang von 1,5\% verglichen mit Januar und Februar des Vorjahres. Die Rahmenvorgaben für Arzneimittel von KBV und GKV-Spitzenverband sehen vor, dass die Ausgaben über das Jahr 2011 gesehen um 0,2\% steigen. Im Gegensatz zu den Ausgaben hat die Menge der abgegebenen Packungen zugenommen: um 2,9\% auf 55,3 Millionen. Im Januar hatte der Anstieg - wie häufig zu Quartalsbeginn - mit 6,3\% noch höher gelegen.

Wie schon in den vergangenen Monaten dämpfen die Sparinstrumente der jüngsten Gesundheitsreform die Ausgabenentwicklung. So gilt seit August 2010 ein von $6 \%$ auf 16\% erhöhter Zwangsrabatt für Arzneimittel ohne Festbetrag. Im Januar und Februar 2011 hat das die Kassen bereits um 363 Mio. Euro entlastet (Vorjahreszeitraum: 148 Mio. Euro). Seit Jahresbeginn ist nun auch der Apothekenrabatt für rezeptpflichtige Präparate von 1,75 auf 2,05 Euro erhöht worden. Als Folge mussten Apotheken den Kassen Nachlässe in Höhe von 203 Mio. Euro einräumen - 37 Mio. Euro mehr als im Vorjahreszeitraum.

fst

\section{Naturheilkunde \\ Phyto-Forum: Experten beraten online}

—_Gegen alles ist ein Kraut gewachsen", sagt der Volksmund. Das macht deutlich: Pflanzen und Kräuter spielen in der Heilkunst seit Jahrhunderten eine wichtige Rolle. Und auch heute haben pflanzliche Arzneien einen festen Platz in der Pharmakotherapie. Mit dem Online-Forum zur Phytotherapie bietet die „Ärzte Zeitung“ einen neuen Service. Unter www.aerztezeitung.de können sich Angehörige medizinischer Fachkreise kostenlos mit Fragen an Experten wenden. Was kann Phytotherapie leisten? Wo liegen ihre Grenzen? Worauf ב beruht die pharmakologische Wirkung pflanz¿ licher Präparate? Auf welche Wechselwirkungen : ist zu achten? Wo haben sich pflanzliche Arzneimittel besonders bewährt? Zu Phytotherapeutika gibt es immer wieder Fragen - von den Ärzten selbst oder von den Patienten an ihre Ärzte. Diese werden nun im Phyto-Forum von fünf Mitgliedern der Gesellschaft für Phytotherapie beantwortet. red

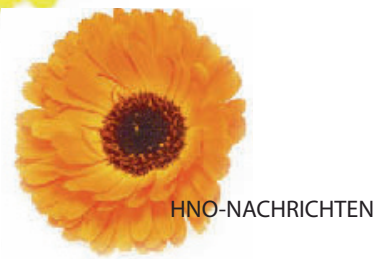

